\title{
Exploring the Dark Continent with fibre Bragg gratings
}

\author{
John W. Arkwright ${ }^{1, *}$, David Hsiao-Chuan Wang ${ }^{1}$, Simon A. Maunder ${ }^{1}$, Neil G. Blenman ${ }^{1}$, Ian \\ Underhill $^{2}$, Vicki Patton ${ }^{3}$, Phil G. Dinning ${ }^{4}$, \\ ${ }^{1}$ CSIRO Material Science and Engineering, PO BOX 218, Lindfield, NSW 2070, Australia \\ ${ }^{2}$ Griffith School of Engineering, Griffith University, Southport, QLD 4222, Australia \\ ${ }^{3}$ Department of Ano-rectal Physiology, St George Hospital, University of new South Wales, NSW \\ 2217, Australia \\ ${ }^{4}$ Dept. Human Physiology, School of Medicine, Flinders University SA 5042, Australia \\ *john.arkwright@csiro.au; phone 612 94137064; www.csiro.au
}

\begin{abstract}
The lower gastrointestinal tract has been referred to as the 'Dark Continent' of the human body because it is so hard to access without resorting to a surgeon's blade. In response to an unmet clinical need we have developed a fibre optic manometry catheter that is now in clinical use across Australia and New Zealand. The unparalleled detail of colonic activity that these devices provide is being hailed as ground breaking by global experts. In this paper we present the design and clinical application of the catheters, and also some of the (sometimes surprising) requirements of our clinical colleagues.
\end{abstract}

Keywords: Fiber optic sensing; Fiber Bragg gratings; Manometry; Colonic motility; Functional gastrointestinal disorders.

\section{INTRODUCTION}

In 2008, in response to a request from a local gastroenterology research group, our team developed a novel optical fibre manometry catheter that was long and flexible enough to span the full length of the human colon [1, 2]. The catheter was based on closely spaced Draw Tower Gratings [3] which enabled us to fabricate mechanically robust catheters that could withstand the everyday rigors of clinical use.

Since 2009 we have been collaborating closely with St George Hospital in Sydney, and Flinders University in Adelaide developing and using these catheters in a series of studies on colonic motility (motility is defined as the ability to move spontaneously, and in GI terms this refers to the movement of material through the gut). The studies reported here were conducted on a total of 49 different subjects from four patient groups (Slow transit constipation, $\mathrm{N}=19$; fecal incontinence, $\mathrm{N}=11$, irritable bowel syndrome, $\mathrm{N}=3$; diverticulitis, $\mathrm{N}=3$ ) and also one control group of healthy subjects $(\mathrm{N}=13)$. The results from some of these studies have been reported at a number of global conferences on gastroenterology and the outcomes of the studies have recently been published in medical journals [4, 5]. The major difference between the fibre optic catheters and more conventional colonic catheters is the number and spacing of the sensing regions that can be incorporated along the sensing array. Conventional catheter technologies have used limited numbers of sensing elements to record from the colon, with sensor spacings ranging from $7.5 \mathrm{~cm}$ to $20 \mathrm{~cm}$. A recently developed solid state catheter has increased the sensor count to 40 with $2.5 \mathrm{~cm}$ spacing between each element which represents the state of the art for conventional manometry devices. The limitation to extending the sensor count has been the decrease in flexibility and increase in complexity that goes with the additional sensing elements. Our fibre optic device uses wavelength division multiplexing (WDM) to address multiple sensing regions along a single fibre length, with up to 36 elements being readily achievable on each fibre, so we are able to fabricate catheters with up to 144 sensing regions using off the shelf FBG interrogators without compromising the flexibility or increasing the overall diameter of the catheters. We now routinely fabricate catheters with $72-120$ elements depending on the patient type and clinical need. Since our first presentation on the results from the studies it was clear that the data from the fibre optic devices far exceeded that of previous colonic motility studies and that we had the potential to generate greater understanding of how this complex organ works. 
In this paper we briefly describe the catheter design and of the clinical trial protocols, then provide details of the increase in recorded data from our devices compared to the current clinical standard. In the conclusion we describe the relevance and perceived benefit of the increased level of information and summarize the advantages that using an FBG based approach has given.

\section{CATHETER DESIGN}

The basic design of the fibre optic catheter has been reported elsewhere [1, 2] and was also presented at this conference in 2008 [6]. In brief, each FBG element in the DTG array was bonded to a rigid substrate and then covered with an elastomeric diaphragm that distorts when the local ambient pressure varies, shown in Figure 1. This distortion pushed the fibre sideways and changes the Bragg wavelength of the FBG. Typically, two separate fibres are used to form a sensing array, with the second fibre being used either to compensate for changes in temperature (by having two FBG elements located at each substrate) or to extend the overall sensing range. For colonic investigations when the device is held at core body temperature, we use the latter approach. Arranging the fibres so that they cross over each other between each substrate ensures that the overall catheter is flexible enough for intubation into the convoluted regions of the gut below the stomach. If more than 72 elements are needed then additional fibres can be threaded through the outer sections of the substrate to form further pressure sensing arrays attached to the distal end of the primary array.
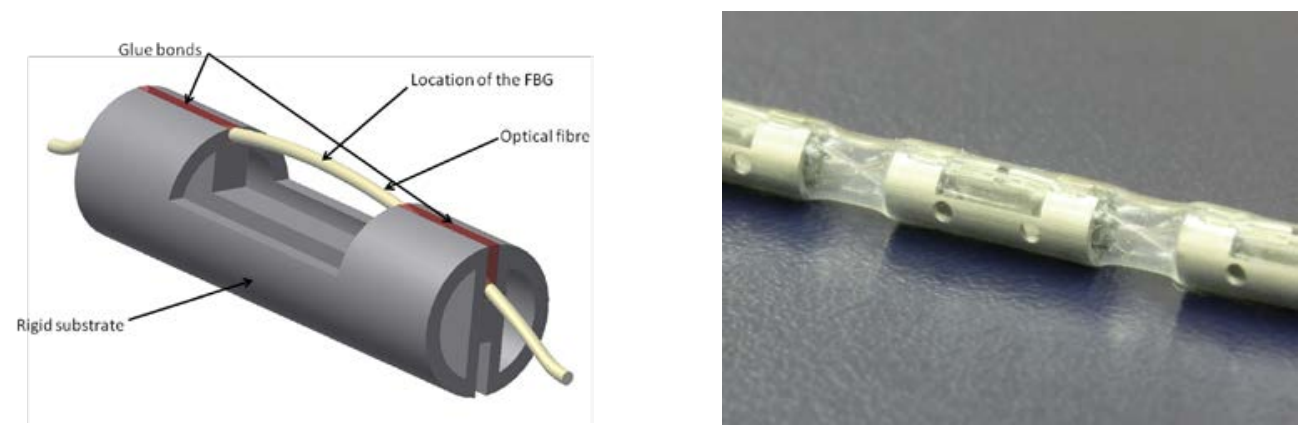

Figure 1. (a) Schematic of the fibre optic pressure sensor. The FBG is located in the curved section of fibre between the two glue bonds. (b) The structure is covered with an elastomeric sleeve to protect the fibre and to create a pressure sensitive diaphragm.

\section{CLINICAL STUDIES}

The clinical studies reported in this presentation were carried out at St George Hospital, Kogarah, NSW, and Flinders Medical Centre in South Australia. The studies were approved by the Human Ethics Committees of the South Eastern Area Health Service, Sydney and the University of New South Wales (05/122, HREC/09/STG/107) and the South Adelaide Clinical Human Research Ethics Committee at Flinders Medical Centre (419.10), and all participants in the trials had given written, informed consent. The catheters were placed using a colonoscope with the tip of the catheter being located as close as possible to the caecum. The full bowel preparation and placement has been described in detail previously. The studies were conducted over periods ranging from 6 hours to $2 \frac{1}{2}$ days depending on the specific study being conducted. The data reported here was gathered from each subject during a selected 2 hour period after recovery from sedation in which significant colonic activity was recorded.

\section{RESULTS}

To compare the fibre optic data to that obtainable using conventional colonic catheters the data from each recording was sub-sampled using every 2nd, 3rd, 5th and 10th sensor to simulate catheters with sensors every 2, 3, 5, and $10 \mathrm{~cm}$. A two hour recording from each data set was then analysed, at each of these sensor spacings, to determine the number of propagating events that traveled in an anal (antegrade) or oral (retrograde) direction. These data were then compared to the full data set at $1 \mathrm{~cm}$ spacing. It was immediately obvious that increasing the sensor spacing had a dramatic effect on the number and even the direction of identified events. Figure 2 shows data from the same section of one of the recordings shown at both 10 and $1 \mathrm{~cm}$ spacing. At $10 \mathrm{~cm}$ there appear to be two antegrade propagating events indicated 
by the dashed red arrows. However, the full data set reveals that these events are in fact made up of a sequence of multiple retrograde events. This example is typical of the confusion in data interpretation that occurs when using $10 \mathrm{~cm}$ spaced data. Figure 3 shows a summary of the number of retrograde and antegrade events identified using each sensor spacing and shows that even reducing the spacing to $2 \mathrm{~cm}$ results in almost 50\% of all propagating events being missed or misinterpreted. At $10 \mathrm{~cm}$ spacing almost $77 \%$ of all antegrade events and $97 \%$ of retrograde events are missed.
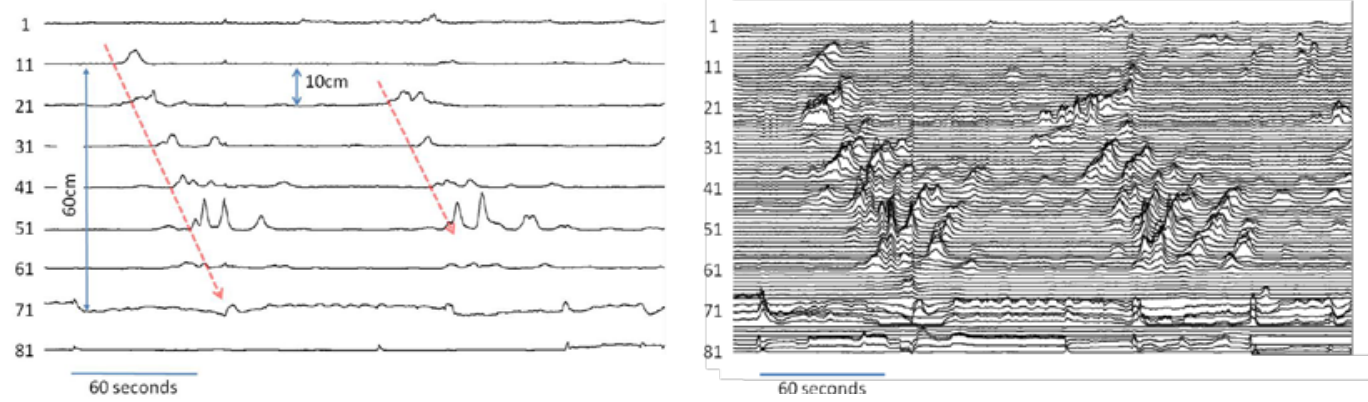

Figure 2. Data recorded from the human colon of a patient suffering from fecal incontinence using a high resolution fibre optic manometry catheter showing (a) responses from every $10^{\text {th }}$ sensor to indicate the output that would be obtained using a traditional colonic catheter with $10 \mathrm{~cm}$ sensor spacing, and (b) responses from the full data set.

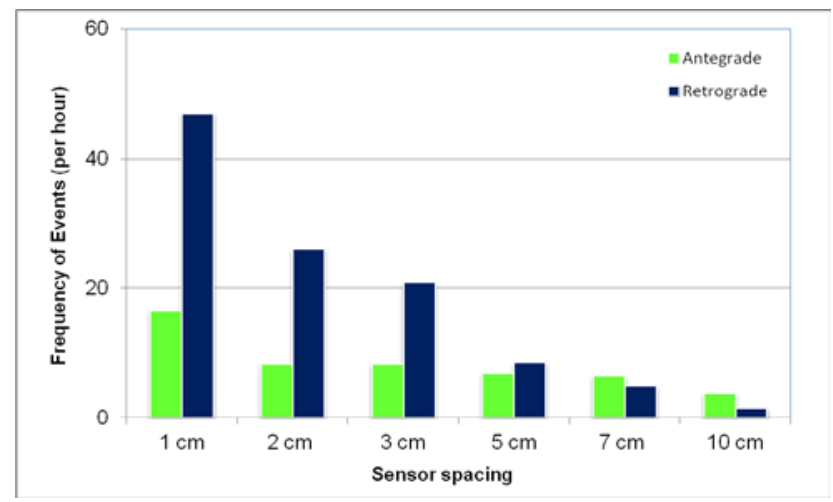

Figure 3. Summary of antegrade and retrograde events identified using different sensor spacings. At $10 \mathrm{~cm}$ spacing almost $77 \%$ of all antegrade events and $97 \%$ of retrograde events identified at $1 \mathrm{~cm}$ spacing are missed

\section{CLINICAL RELEVANCE}

The human GI tract is 5-7 metres in length, extending from the mouth to the anus, and the normal processes of digestion requires the appropriate propulsion (or motility) of contents controlled by sequences of muscular contractions. The key feature of normal motility is that it involves patterns of contraction and relaxation, coordinated over considerable lengths of gut, over periods of seconds to minutes, all hidden deep within the body. This makes it difficult to record and quantify motility events using established technologies. While most of us can take normal gut motility for granted, dysmotility in children and adults can cause significant personal, societal and financial burdens, costing the healthcare systems many billions of dollars per year [7]. In the colon, motility disorders such as constipation, faecal incontinence and irritable bowel syndrome (IBS) are very common $[8,9]$ and for many patients with these conditions the treatment options remain poor. One of the main problems is that we have a very incomplete understanding of the mechanical processes that underpin these conditions. In 1908, Hertz published a paper on the treatment of constipation which opened: “The treatment of chronic constipation is always experimental. One method is tried after another until by chance that suited to the individual case is discovered" [10]. Today, very little has changed, and this is possibly due to the limitations of traditional diagnostic methods. The ability of optical fibre to record contractile events along physiologically significant lengths of the GI tract with high enough spatial resolution may provide one of the causal links between motility and symptoms, and hence lead to improved therapeutic interventions for sufferers of these disorders. 


\section{IMPLICATIONS FOR FIBRE SENSING TECHNOLOGIES}

These studies serve as a clear indication of the benefits of using optical fibre sensing techniques for clinical in-vivo measurement. The use of draw tower gratings has made it possible to fabricate FBG based devices that are robust and flexible enough for routine clinical use. The use of FBGs with WDM techniques makes it possible to interrogate large numbers of sensing elements along a single fibre using a single spectral detector. This has significant benefits when monitoring parameters along physiologically significant regions of the body such as the gastrointestinal tract, the cardiovascular and pulmonary systems. Although not discussed in this work, optical fibre based devices are also a perfect sensing mechanism for in-vivo monitoring within magnetic resonance imaging equipment.

\section{CONCLUSION}

Recently developed fibre optic manometry catheters have been used in a series of clinical trials studying motility in the human colon. The results have demonstrated the benefit of using FBGs and WDM techniques to monitor pressures at multiple closely spaced locations along the human gastrointestinal tract. The increased levels of information available from the fibre optic catheters are revolutionizing our knowledge of the normal contractile patterns of the healthy human colon and importantly giving us insight into the motor patterns associated with colonic disorders.

\section{REFERENCES}

[1] J. W. Arkwright, N. G. Blenman, I. D. Underhill et al., "In-vivo demonstration of a high resolution optical fiber manometry catheter for diagnosis of gastrointestinal motility disorders,” Optics Express, 17(6), 4500-4508 (2009).

[2] J. W. Arkwright, I. D. Underhill, S. A. Maunder et al., "Design of a high-sensor count fibre optic manometry catheter for in-vivo colonic diagnostics,” Optics Express, 17(25), 22423-22431 (2009).

[3] E. Lindner, J. Morbitz, C. Chojetzki et al., "Draw Tower Fiber Bragg Gratings and their use in sensing technology,” Fiber Optic Sensors and Applications Viii, 8028, (2011).

[4] P. G. Dinning, L. M. Hunt, J. W. Arkwright et al., "Pancolonic motor response to subsensory and suprasensory sacral nerve stimulation in patients with slow-transit constipation,” Br J Surg, 99(7), 1002-10 (2012).

[5] V. Patton, L. Wiklendt, J. W. Arkwright et al., "The effect of sacral nerve stimulation on distal colonic motility in patients with faecal incontinence,” Br J Surg, 100(7), 959-68 (2013).

[6] J. W. Arkwright, S. N. Doe, M. C. Smith et al., "Design and clinical results from a fibre optic manometry catheter for oesophageal motility studies - art. no. 70042D,” 19th International Conference on Optical Fibre Sensors, Pts 1 and 2, 7004, D42-D42 (2008).

[7] J. E. Everhart, and C. E. Ruhl, "Burden of digestive diseases in the United States part I: overall and upper gastrointestinal diseases,” Gastroenterology, 136(2), 376-86 (2009).

[8] A. E. Bharucha, A. R. Zinsmeister, G. R. Locke et al., "Prevalence and burden of fecal incontinence: a population-based study in women,” Gastroenterology, 129(1), 42-9 (2005).

[9] I. J. Cook, N. J. Talley, M. A. Benninga et al., "Chronic constipation: overview and challenges,” Neurogastroenterol Motil, 21 Suppl 2, 1-8 (2009).

[10] A. F. Hertz, "The Pathology and Treatment of Chronic Constipation," Proc R Soc Med, 1(Med Sect), 119-49 (1908). 